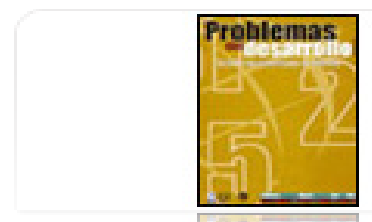

Problemas del Desarrollo. Revista Latinoamericana de Economía

ISSN: 0301-7036

revprode@servidor.unam.mx

Universidad Nacional Autónoma de México

México

Delgadillo Macías, Javier

Dimensiones territoriales del desarrollo rural en América Latina

Problemas del Desarrollo. Revista Latinoamericana de Economía, vol. 37, núm. 144, enero-marzo,

2006, pp. 97-120

Universidad Nacional Autónoma de México

Distrito Federal, México

Disponible en: http://www.redalyc.org/articulo.oa?id=11820097005

Cómo citar el artículo

Número completo

- Más información del artículo

Página de la revista en redalyc.org

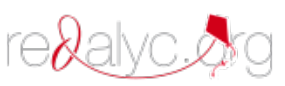

Sistema de Información Científica

Red de Revistas Científicas de América Latina, el Caribe, España y Portugal

Proyecto académico sin fines de lucro, desarrollado bajo la iniciativa de acceso abierto 


\section{DIMENSIONES TERRITORIALES DEL DESARROLLO RURAL en AmÉRICA LATINA*}

\section{Javier Delgadillo Macías**}

Fecha de recepción: 27 de octubre de 2005. Fecha de aceptación: 30 de enero de 2006.

\section{Resumen}

Se ve al desarrollo rural como un proceso de organización y modificación integral de factores que interactúan al interior de lugares y territorios determinados y que afecta de manera directa o indirecta aspectos culturales, ambientales, económicos y sociales propios de los habitantes y de la vida de cada ámbito rural. Su conceptuación y los alcances de las políticas públicas emprendidas a su favor han pasado por diversidad de enfoques y aplicaciones con un común denominador: sobredimensionar por cualquier otro aspecto el carácter sectorial. Hoy, las estrategias y acciones para hacer relevante la vida rural en los países latinoamericanos deben centrar su atención en un esfuerzo por combinar un enfoque de desarrollo desde la visión del territorio como sujeto de acción — política e institucional-, desde el cual se promueva la constitución de una nueva institucionalidad proactiva que beneficie al campo y a la población que los habita.

Palabras clave: desarrollo territorial, desarrollo rural, microrregiones, nueva institucionalidad, competitividad sistémica.

* El autor agradece a Ricardo Méndez, del Instituto de Economía y Geografía del Consejo Superior de Investigaciones Científicas de Madrid, y a Rafael Echeverri, consultor del Instituto Interamericano de Cooperación para la Agricultura, la lectura y sus muy importantes recomendaciones al texto. También las opiniones y criterios técnicos de los dictaminadores anónimos.

** Investigador titular del Instituto de Investigaciones Económicas de la UNAM, coordinador del Programa de Estudios Regionales en el Centro Regional de Investigaciones Multidisciplinarias de la UNAM. Profesor invitado de El Colegio de Tlaxcala, A.C. Miembro del Sistema Nacional de Investigadores (SNI). Correo electrónico: jdm@servidor.unam.mx. 


\begin{abstract}
Rural development is seen as process of organization and integral modification of factors that interact within determined locations and territories and which directly or indirectly affect the cultural, environmental, economic and social aspects of the inhabitants themselves and the life of each rural area. Its conceptualization and the scope of public policies unfolding in its favor have gone through many focuses and applications with a common denominator: the sectoral character standing out against all other aspects. Today, the strategies and actions to make rural life relevant in the Latin American countries should be centered on efforts to combine a development focus from the viewpoint that the territory is the subject of political and institutional action. In this way, the establishment of a new pro-active institutionalism will be promoted to benefit the countryside and the people who live in it.
\end{abstract}

Key words: territorial development, rural development, micro-regions, new institutionalism, systemic competitiveness.

\title{
Résumé
}

On considère le développement rural comme un processus d'organisation et de modification intégrale de facteurs qui agissent entre eux à l'intérieur de lieux et de territoires déterminés et qui touche de manière directe ou indirecte des aspects culturels, environnementaux, économiques et sociaux propres des habitants et de la vie de chaque environnement rural. Sa conceptualisation et les atteintes des politiques publiques entreprises en sa faveur sont passées par une diversité de points de vues et d'applications ayant toutes un facteur commun: surdimensionner le caractère sectoriel par dessus tous les aspects. Aujourd'hui, les stratégies et les actions utilisées pour donner du relief à la vie rurale dans les pays latino-américains doivent centrer leur attention sur un effort pour combiner un point de vue de développement depuis la vision du territoire comme sujet d'action — politique et institutionnel一, à partir duquel on promeut la constitution d'une nouvelle institutionnalité proactive qui bénéficie la campagne et la population qui y habite.

Mots-cléfs: développement territorial, développement rural, micro régions, nouvelle institutionnalité, compétitivité systémique.

\section{Resumo}

O desenvolvimento rural é percebido como um processo de organização e modificação de fatores que interatuam no interior de lugares e territórios determinados e que afeta de maneira direta ou indireta aspectos culturais, ambientais, económicos e sociais próprios dos moradores e da vida de cada âmbito rural. A sua conceituação e os logramentos das políticas públicas empreendidas ao seu favor passaram por diversidade de enfoques e aplicações com um comum denominador: sobre-dimensionar por qualquer outro aspecto o caráter setorial. Hoje, as estratégias e ações para fazer relevante a vida rural nos países da América Latina, devem centrar sua atenção no esforço por harmonizar um enfoque de desenvolvimento desde a visão de território como sujeito de ação — política e institucional - desde o qual possa se promover a constituição duma nova institucionalidade pró-ativa que beneficie ao campo e à população que habita-os.

Palavras chave: desenvolvimento territorial desenvolvimento rural, microrregiões, nova institucionalidade, competitividade sistêmica.

\section{Desarrollo}




\section{Presentación}

$\mathrm{E}$

1 desarrollo rural es visto como un proceso de organización y modificación inte-

gral de factores que interactúan al interior de lugares y territorios determinados y

que afecta de manera directa o indirecta aspectos culturales, ambientales, económicos y sociales propios de los habitantes y de la vida de cada ámbito rural. Su conceptuación y los alcances que las políticas públicas emprendidas a su favor pasan por diversidad de enfoques y aplicaciones con un común denominador: sobredimensionar por cualquier otro aspecto el carácter sectorial. Sin embargo, las estrategias y acciones para hacer relevante la vida rural al interior de nuestros países requieren poner énfasis en combinar un enfoque de desarrollo rural desde la visión del territorio como sujeto de acción — que promueva una nueva institucionalidad proactiva en beneficio del medio rural y de las actividades que en éste se desarrollan-, considerando, entre otros factores, las actividades no agrícolas emergentes, pero, sobre todo, tomando como centro de atención los propios lugares y a la población que los habita. A partir de estas consideraciones propias del enfoque territorial del desarrollo rural, el presente artículo tiene como objetivo principal presentar los postulados básicos de ese enfoque de políticas públicas que en los últimos años se viene proponiendo, y dimensionar a partir de experiencias directas en México $^{1}$ y documentales de otros países latinoamericanos, su posible instrumentación.

\section{Introducción}

América Latina está inmersa en procesos de cambio de gran relevancia para la vida rural. Frente a esos retos, el desafío se centra en las capacidades de cada país para absorber, en su interior y de manera positiva, dichos cambios impulsados por factores internos e internacionales acumulados, gran parte de ellos a lo largo del tiempo, y otros cuya presencia se da en pocos años. Lo anterior se hace evidente cuando observamos cómo los territorios rurales incorporan una importante y creciente economía no agrícola, que demanda cerca de $40 \%$ de la mano de obra del campo y la mitad del ingreso de sus pobladores.

1 La Universidad Nacional Autónoma de México, mediante su Centro Regional de Investigaciones Multidisciplinarias y del Instituto de Investigaciones Económicas, viene realizando un proyecto aplicado de desarrollo rural territorial y coordina la Red de Investigación Socioregional e Información Georeferenciada de México. A partir de esas instituciones se han generado diversas experiencias de vinculación con grupos de investigación de las universidades de los estados de Morelos, Tlaxcala, Guerrero, Oaxaca y Chiapas, en ámbitos comunitarios y microrregionales. Experiencias derivadas de este grupo pueden revisarse en los libros El desarrollo territorial en Tlaxcala: dimensiones rurales y opciones de ordenamiento espacial en la Región Oriente (Delgadillo, 2005) y Enfoque territorial para el desarrollo rural en México (Delgadillo, 2006).

\section{DeSarrollo}


Sin embargo, para transitar por esos nuevos derroteros, los países requieren afrontar, de manera decidida, problemas sociales y económicos acendrados en sus territorios rurales, producto de los desiguales términos de intercambio que han favorecido, en la mayoría de los casos, a los entornos urbanos.

En términos poblacionales, al inicio del presente siglo América Latina registró una población de 503.1 millones de habitantes aproximadamente, ${ }^{2}$ de los cuales $25 \%$, es decir, 125.78 millones, residía en zonas rurales, con una tasa de crecimiento promedio anual estable, en niveles de $0.1 \%$ para el periodo de 1981-2000, distinguiéndose los países de América Central con la mayor velocidad de crecimiento (1.8\% anual) y la región sur con $-0.7 \%$ en ese lapso (CEPAL, 2004).

Aunque el ritmo de crecimiento de la población rural en la década 1990-2000 mantuvo niveles estables bajos, la participación de ésta se redujo de $29.3 \%$ a $25.0 \%$, debido al aumento de la población localizada en las grandes ciudades y ciudades medias, principalmente, como resultado de los intensos procesos migratorios que se iniciaron casi al comienzo del siglo pasado y que incrementaron sustancialmente los grados de urbanización, con la consecuente formación de cinturones de miseria, proliferación de la informalidad y polarización social.

El marco de esos nuevos escenarios se deriva básicamente del proceso de apertura comercial que afecta al conjunto de países y sus regiones, caracterizado por la desgravación arancelaria, los acuerdos de libre comercio y los compromisos multilaterales adquiridos en la Organización Mundial del Comercio, los cuales han establecido límites restringidos a los centros de toma de decisión de la agricultura y de la vida rural. A su vez, la liberalización de los mercados no sólo ha minimizado la intervención estatal, que buscaba alterar las condiciones de equilibrio en aras de la equidad, sino que ha marginado casi por completo las iniciativas sectoriales y territoriales al privilegiar los balances macroeconómicos (Trejos et al., 2004). En la situación actual, muchos de los instrumentos empleados en el pasado ya no pueden utilizarse o se aplican con mucha menor intensidad. Esto es así porque la liberalización económica les hace perder legitimidad debido a que los países siguen ahora una estrategia de desarrollo diferente o participan en procesos de integración que los obligan a armonizar políticas, y también porque se han establecido reglas y acuerdos de comercio internacional que lo impiden.

El empleo tradicional y los mecanismos de respuesta social e institucional que antes cobijaban los desajustes en la estructura productiva del campo, hoy conviven con procesos y dinámicas organizacionales y productivas que no acaban de asentarse en territorios al interior de los países latinoamericanos, debido, entre otros factores, a la dualidad entre modernidad y tradición, que si bien debieran ser incluyentes, derivan en un lento camino de integración social y territorial de las sociedades rurales.

2 El Anuario Estadístico de América Latina y el Caribe (2004) de la CEPAL estima para 2005 una población total de 590.4 millones.

\section{DeSarrollo}




\section{El territorio como sujeto del desarrollo}

Como ya hemos mencionado, un denominador común en las políticas de atención al medio rural ha sido dimensionar su aspecto sectorial dentro de la dinámica general del desarrollo de un país o una región. Según Rodríguez (2004), es posible establecer una clasificación de las diferentes perspectivas que adoptan los países, tanto en relación con el momento temporal concreto y el espacio de referencia, como de la línea sectorial dominante. En la sucesión de enfoques y paradigmas se identifica, en los últimos años, un efecto moda, en el sentido de que la alternancia de opciones y estrategias implica, en ocasiones, la adopción de principios difícilmente complementarios entre sí. De acuerdo con ese autor, las primeras intervenciones incidieron de modo casi exclusivo en el incremento de la capacidad productiva agraria; posteriormente, éstas se verán relegadas por una línea de trabajo de marcado carácter sociocultural; finalmente, se adopta un papel centrado en la multiplicidad de enfoques relacionados con la sostenibilidad (sustentabilidad) y el desarrollo humano y territorial.

En esta última corriente se sitúa el enfoque territorial del desarrollo rural, el cual, como señalan Sepúlveda et al. (2003b), es apenas el estadio lógico de un largo proceso de aproximaciones a un nuevo paradigma para América Latina. Entre las más actuales se puede citar la reforma agraria de la década de 1960, el cooperativismo de los setenta, la modernización productiva impulsada por la revolución verde de los años sesenta y setenta, el desarrollo rural integrado de los ochenta, la modernización y la vinculación comercial a mercados dinámicos y el desarrollo sostenible de los años noventa, así como la participación y el empoderamiento de los pobladores rurales, impulsados en fechas más recientes.

La sostenibilidad de los medios de vida, la cooperación y la gobernabilidad locales, el fortalecimiento de una nueva economía basada en la agricultura y la incorporación de un concepto ampliado de economía territorial, y los riesgos y oportunidades que supone la globalización para la viabilidad de las economías rurales son algunas de las preocupaciones que abarca el enfoque territorial, cuyo surgimiento se da en respuesta a una serie de factores que afectan el proceso de desarrollo rural, entre ellos:

a) la persistencia de la pobreza rural;

b) la insuficiencia de los ajustes y las reformas estructurales para solucionar los desafíos básicos del desarrollo;

c) la persistencia de brechas regionales y sectoriales;

d) el sesgo antirural de los esquemas de provisión de infraestructura;

e) la inseguridad alimentaria que padecen las poblaciones rurales;

f) la importancia estratégica que continúa teniendo la agricultura en muchas economías de la región;

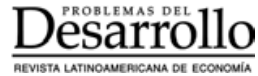


g) la importancia creciente de las actividades no agrícolas en el ámbito rural y la expansión de centros urbanos en territorios mayormente rurales;

h) el deterioro de los recursos naturales;

i) la transformación que ha experimentado la institucionalidad rural;

j) la evolución de las expectativas de los consumidores y los mercados; y

k) el auge de las nuevas tecnologías de información y comunicación (Sepúlveda et al., 2003a).

El enfoque territorial del desarrollo rural es una propuesta centrada en los puntos de interacción entre los sistemas humanos y los ambientales, a partir de la cual se impulse la integración de los sistemas productivos propios del campo y se genere el bienestar y la inclusión del mayor número posible de grupos sociales relegados. Desde esta perspectiva, los territorios rurales se definen como espacios geográficos cuya cohesión deriva de un tejido social específico, de una base de recursos naturales particular, de instituciones y formas de organización propias, y de determinadas formas de producción, intercambio y distribución del ingreso que les dan especificidad regional. En esa concepción, el territorio sirve como elemento integrador de agentes, mercados y políticas públicas, al mismo tiempo de ser el objetivo del desarrollo económico.

Las formas tradicionales en las cuales el campo se relacionaba con las ciudades fueron, y aún lo son en muchos casos, procesos para el envío de bienes primarios y recursos naturales, intensas migraciones de origen rural y como oferentes de servicios turísticos paisajísticos a visitantes de las áreas urbanas. Por su parte, las ciudades suministran a las áreas rurales bienes manufacturados, devolviéndoles parte de sus bienes primarios y recursos naturales, pero con valor agregado; les provee insumos industriales para la producción agrícola y otro tipo de producción primaria; acoge a la población migrante y los ubica generalmente en los barrios suburbanos y en sectores informales de subsistencia y, con ello, las ciudades dominan al campo y lo someten a su servicio, proveyéndose de mano de obra barata y funcional. Esta dinámica ha originado la subordinación creciente del campo y la polarización territorial expresada en los muy diferentes grados de concentración y crecimiento territoriales. También ha logrado acentuar las divergencias sociales en términos de ingresos y acceso a los servicios públicos, así como mantener correspondencia con las pronunciadas diferencias económico-productivas de las estructuras sectoriales de los países de la región.

Frente a esas relaciones asimétricas y un incremento de problemas para la subsistencia de la ruralidad en los últimos años, se han sugerido estrategias y acciones para redimensionar la vida rural en los países latinoamericanos, ${ }^{3}$ medidas que, en gran número de ocasiones,

3 La difusión de este pensamiento cada vez se incorpora más en el ámbito de la esfera pública de los países de América Latina, e incluso está presente en diversas propuestas del Banco Interamericano de Desarrollo (BID), del Banco Mundial, de la CEPAL, del Instituto Interamericano de Cooperación

\section{DeSarrollo}


centran su atención en el esfuerzo por combinar un enfoque de desarrollo rural desde la visión del territorio como sujeto de acción política e institucional, y que promuevan la construcción de una nueva institucionalidad proactiva a partir de la visión holística, multidimensional, intertemporal y multisectorial de la articulación territorial. Sin embargo, no es suficiente formular orientaciones particulares y promover políticas diferenciadas, si no se establece un compromiso claro y decidido de fortalecimiento del sector agrícola como factor productivo clave del mundo rural y se fortalece, a la vez, la presencia de agentes y dimensiones que lo posibiliten y que ponderen la importancia de la competitividad sistémica como instrumento que lo hace viable. Nos referimos a un término de competitividad productiva y comercial que asume las ventajas a partir de un conjunto de atributos que un país, región, estado, municipio, ciudad o territorio rural posee, el cual posibilita a las empresas localizadas minimizar sus costos de ubicación y operación para colocar sus productos en los mercados nacionales e internacionales a un precio y calidad competitivos. Pero más allá de lo estrictamente económico, la competitividad del orden territorial alude a un concepto mucho más amplio, que incorpora la multidimensionalidad de factores y procesos y pone en el centro de la atención pública a los actores sociales residentes en el territorio o el sistema territorial identificado. El concepto competitividad sistémica territorial reconoce que la competitividad de un determinado ámbito es el resultado de la acción conjunta de una serie muy amplia de factores públicos, privados, sociales y personales que actúan de forma simultánea en un territorio determinado (aregional.com, 2005). ${ }^{4}$

para la Agricultura (IICA) y demás organizaciones, que plantean hoy con insistencia promover el desarrollo rural con enfoques amplios, territoriales y multisectoriales. Así, estos organismos señalan que el campo comprende gran variedad de actividades económicas (agropecuarias, forestales, pesque ras, agroindustriales y agroalimentarias, minería, energía, agroturismo y otras), junto a acciones que favorecen las áreas de educación y salud, infraestructura, transporte, y actividades financieras, entre otras.

4 El concepto competitividad sistémica considera que las inversiones captadas por una región deben traducirse en mayores niveles de empleo, ingresos y, por tanto, bienestar. Para ello, se requiere que el marco de actuación de la empresa sea dentro de un grupo empresarial organizado en redes de colaboración en las cuales se intercambien insumos, tanto en sentido físico y tradicional (materias primas y materiales), como aquellos intangibles, como información, tecnología, habilidades y conocimientos, elementos fundamentales que permiten generar importantes ahorros en costos dentro del proceso productivo (o, lo que es lo mismo, rendimientos crecientes) en el agregado territorial. Por este motivo, el entorno global debe facilitar la interacción dinámica de la capacidad organizativa de las empresas con la sociedad, el Estado y las instituciones intermedias; es decir, de forma conjunta, armónica y congruente entre distintos niveles de un sistema nacional: desde el micro persona, el micro regional, hasta el meta e internacional. Competitividad sistémica tiene su origen en el Instituto Alemán de Desarrollo (German Development Institute, GDI por sus siglas en inglés), al cual se le atribuye una amplia serie de trabajos relacionados con el tema a escala in ternacional. Véase, por ejemplo, Esser et al., "Competitividad sistémica: Competitividad internacional de las empresas y políticas requeridas”, Berlín, 1994.

\section{DeSarrollo}


El tiempo transcurrido parece darle la razón a este enfoque: hoy encontramos respuestas al interior de los países y eco de parte de los organismos internacionales corresponsables de consolidar este viraje a favor de la equidad territorial.

\section{¿Qué tanto se ha avanzado en la integración social e institucional de los territorios rurales?}

La situación de pobreza de un importante sector de población que hoy habita los espacios rurales tiene trascendental incidencia en la estructura social del conjunto de la población, distribuyéndose espacialmente la marginación al constatar que más de la mitad de sus hogares se enfrentan a condiciones de pobreza, lo que da origen también a la pobreza urbana.

Los expandidos niveles de pobreza y pobreza extrema rural, asociadas a los procesos de desintegración social, se convierten en serias limitantes a toda posibilidad de reactivar territorios productivos, al tiempo que socavan procesos identitarios y de concertación básicos en el desarrollo territorial rural, tal como lo establecen Schejtman y Berdegué (2003), para quienes el territorio es un espacio con identidad y con un proyecto de desarrollo concertado socialmente, y donde cada proceso de desarrollo es (debe ser) una construcción social y no un espacio objetivamente existente en virtud (sólo) de variables físicas o económicas.

Los indicadores sociales son más que elocuentes: más de $60 \%$ de la población rural de países como Nicaragua, Bolivia, Guatemala y Honduras se ubican por debajo de la línea de pobreza; otras naciones, como México y Perú, presentan también proporciones altas, entre 50 y $60 \%$ (Cuadro 1). Si bien esos países se ven beneficiados por los flujos de remesas de la población radicada básicamente en Estados Unidos, habría que señalar que los recursos que ingresan a las comunidades rurales sólo sirven de paliativos a sus graves problemas sociales y de desintegración familiar.

Por otra parte, contrario al supuesto recurrente en las políticas públicas de América Latina, debemos partir de la consideración de que el desarrollo rural es un proceso social en construcción, asociado hoy a nuevos actores y expresiones culturales y productivas antes inimaginadas. Un proceso al cual se deben incorporar estrategias que fomenten mayor participación, concertación social, alianzas público-privadas, consolidación de redes, en un contexto de impulso a la necesaria articulación de lo rural y lo urbano y con criterios de ordenamiento territorial equilibrado, entendido como un proceso resultante de una planeación social, territorial y productiva de carácter multisectorial y multifuncional para el conjunto de actores locales.

La aceptación en el ámbito de las políticas públicas de que el mundo rural es estructuralmente heterogéneo permitiría, en el mediano plazo, enfocar sistemas de promoción del desarrollo con el criterio de atención diferenciada. Atendiendo a las caracterís-

\section{DeSarrollo}


Cuadro 1

Tasa de crecimiento de las remesas y porcentaje de la población rural en la línea de pobreza (2002-2003). Países seleccionados de América Latina

\begin{tabular}{|c|c|c|c|}
\hline \multirow{2}{*}{$\begin{array}{l}\text { Tasa anual de crecimiento } \\
\text { de las remesas }\end{array}$} & \multicolumn{3}{|c|}{ Población rural bajo la línea de pobreza (\%) } \\
\hline & Más de $60 \%$ & Más de 35\% y menos de 60\% & Menos de $35 \%$ \\
\hline Aumentan más de $100 \%$ & $\begin{array}{l}\text { Nicaragua } \\
\text { Bolivia }\end{array}$ & & Costa Rica \\
\hline Aumentan menos de $100 \%$ & $\begin{array}{l}\text { Guatemala } \\
\text { Honduras } \\
\text { Paraguay }\end{array}$ & $\begin{array}{l}\text { Venezuela } \\
\text { República Dominicana } \\
\text { Colombia } \\
\text { México } \\
\text { Perú } \\
\text { El Salvador } \\
\text { Brasil }\end{array}$ & \\
\hline Disminuyen & & & $\begin{array}{l}\text { Panamá } \\
\text { Chile }\end{array}$ \\
\hline
\end{tabular}

Fuente: UNCTAD (http://www.unctad.org) y CEPAL: Panorama Social de América Latina, 2003-2004.

ticas de las dimensiones ambiental, institucional, social y económica, los indicadores que califican a los países latinoamericanos son, en la mayor proporción, deficitarias de las aspiraciones de sostenibilidad deseadas. En el Cuadro 2 se presentan los índices correspondientes a dichas dimensiones, agrupadas en criterios de sostenibilidad altos, medios y bajos; de los 21 países seleccionados, la mayoría de ellos (16), incluyendo a México, se ubican en niveles medios y bajos, y sólo cuatro (Chile, Uruguay, Argentina y Costa Rica) presentan índices de desarrollo sostenible altos.

Junto a lo anterior, el enfoque de política territorial combinada que aquí se propone deriva también del hecho de que la región latinoamericana, aunque mantiene un fuerte sector agrícola, es predominantemente urbana en términos demográficos, y este fenómeno impacta de manera importante la cultura rural, como consecuencia de su vínculo inevitable con el medio urbano, presentando expectativas y patrones de vida cada vez más semejantes entre los habitantes de ambos territorios, especialmente entre los jóvenes, como lo refieren Schejtman y Berdegué (2003), quienes también documentan cómo hoy la incorporación masiva de las mujeres rurales al mundo del trabajo extraparcelario modifica las relaciones intrafamiliares y los papeles tradicionales de género; la radio y la televisión llegan con sus nuevos mensajes y pautas a todos los rincones del campo; decenas de millones de latinoamericanos rurales han tenido la experiencia de vivir y trabajar en Estados Unidos o en Europa, y muchos de ellos re-gresan a sus regiones de origen con nuevas músicas, nuevas vestimentas, nuevos hábitos de consumo y de recreación, nuevas conciencias de sus derechos ciudadanos, nuevas aptitudes y habilidades.

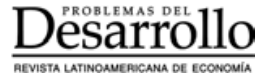


Cuadro 2

América Latina (21 países): heterogeneidad y sostenibilidad según dimensiones por países. Dimensiones e índices

\begin{tabular}{|c|c|c|c|c|}
\hline País & $\begin{array}{c}\text { Dimensión } \\
\text { ambiental } \\
\qquad A M B \\
I S A\end{array}$ & $\begin{array}{c}\text { Dimensión } \\
\text { institucional } \\
\text { INST } \\
\text { IPC }\end{array}$ & $\begin{array}{c}\text { Dimensión } \\
\text { social } \\
\text { SOC } \\
\text { IDH }\end{array}$ & $\begin{array}{c}\text { Dimensión } \\
\text { económica } \\
\text { ECO } \\
I P I B\end{array}$ \\
\hline \multicolumn{5}{|c|}{ Desarrollo sostenible alto } \\
\hline Chile & 55.1 & 74.0 & 83.9 & 77.0 \\
\hline Uruguay & 66.0 & 55.0 & 83.3 & 73.0 \\
\hline Costa Rica & 63.2 & 43.0 & 83.4 & 75.0 \\
\hline Argentina & 61.5 & 25.0 & 85.3 & 78.0 \\
\hline \multicolumn{5}{|c|}{ Desarrollo sostenible medio } \\
\hline Brasil & 59.6 & 39.0 & 77.5 & 73.0 \\
\hline Colombia & 59.1 & 37.0 & 77.3 & 69.0 \\
\hline Panamá & 60.0 & 34.0 & 79.1 & 69.0 \\
\hline México & 45.9 & 36.0 & 80.2 & 75.0 \\
\hline Perú & 56.5 & 37.0 & 75.2 & 65.0 \\
\hline República Dominicana & 48.4 & 33.0 & 73.8 & 70.0 \\
\hline El Salvador & 48.7 & 37.0 & 72.0 & 65.0 \\
\hline Venezuela & 53.0 & 24.0 & 77.8 & 67.0 \\
\hline Ecuador & 54.3 & 22.0 & 73.5 & 60.0 \\
\hline \multicolumn{5}{|c|}{ Desarrollo sostenible bajo } \\
\hline Jamaica & 40.0 & 38.0 & 76.4 & 61.0 \\
\hline Paraguay & 57.8 & 16.0 & 75.1 & 64.0 \\
\hline Guatemala & 49.6 & 24 & 64.9 & 62 \\
\hline Bolivia & 59.4 & 23 & 68.1 & 53.0 \\
\hline Nicaragua & 51.8 & 26.0 & 66.7 & 54.0 \\
\hline Honduras & 53.1 & 23.0 & 67.2 & 54.0 \\
\hline Haití & 34.8 & 15.0 & 46.3 & 46.0 \\
\hline
\end{tabular}

Fuente: elaboración a partir de información del IICA y de: 1) Dimensión ambiental (ISA-Índice de Sostenibilidad Ambiental): Datos del informe Índice de Sostenibilidad Ambiental 2002, publicación del Foro Económico Mundial en colaboración con Yale Center for Environmental Law and Policy (Yale University) y el Center for International Earth Science Information Network (Columbia University). 2) Dimensión institucional (IPC = Índice de Percepción de la Corrupción —cuanto mayor sea el valor del índice menor es la percepción de corrupción): Datos de Transparencia Internacional, 2002. 3) Dimensión social (IDH = Índice de Desarrollo Humano): Datos del Informe de Desarrollo Humano de 2003 (PNUD, 2004). 4) Dimensión económica (IPIB = Índice de Producto Interno Bruto): Datos del Informe de Desarrollo Humano de 2003 (PNUD, 2004).

Ahora bien, si lo vemos por el lado del empleo en agricultura, en América Latina, como porcentaje del empleo total, éste disminuyó en la década de los noventa en relación con finales de los ochenta, pues pasó a representar 18.5\% en el periodo 1991-1999 respecto de 19.7\% en el de 1986-1989; la agricultura sigue siendo una actividad importante dentro de las economías rurales del continente y, en algunas de ellas, sin duda la principal. Junto 
Cuadro 3

Ingreso rural no agrícola como proporción de los ingresos rurales. Países seleccionados de América Latina

\begin{tabular}{lcc}
\hline País & $\begin{array}{c}\text { Año } \\
\text { de la encuesta }\end{array}$ & $\begin{array}{c}\text { Participación del ingreso rural } \\
\text { no agrícola en los ingresos rurales }\end{array}$ \\
\hline Brasil & 1997 & 39 \\
Chile & 1997 & 41 \\
Colombia & 1997 & 50 \\
Costa Rica & 1989 & 59 \\
Ecuador & 1995 & 41 \\
El Salvador & 1995 & 38 \\
Haití & 1996 & 68 \\
Honduras & 1997 & 22 \\
México & 1997 & 55 \\
Nicaragua & 1998 & 42 \\
Panamá & 1997 & 50 \\
Perú & 1997 & 50 \\
\hline
\end{tabular}

Fuente: Reardon, Berdegué y Escobar (2004).

a la importancia del sector agrícola, dentro de las actividades no agrícolas están emergiendo con fuerza el desarrollo de otras labores relevantes que tienen su base en la utilización de los recursos naturales, los cuales determinan su localización territorial, como el turismo (ecoturismo, agroturismo, turismo de playa y otras actividades de recreación sobre la base del uso del paisaje y los recursos naturales), el mercado de servicios ambientales, de artesanías y otros que presentan importantes interrelaciones en determinado territorio, en el cual la separación de lo rural y urbano se torna, muchas veces, un tanto ficticia.

La agricultura primaria, con una localización indiscutible en los territorios rurales, ha presentado en la última década problemas crecientes de rentabilidad relativa (especialmente en las producciones tradicionales). Lo anterior se evidencia en el menor crecimiento de la ocupación en el sector entre 1990 y 2000, comparado con un incremento mayor en los sectores no agrícolas (Dirven, 2004), lo cual, asociado a la expulsión de la fuerza de trabajo ocupada en la agricultura que no es absorbida por el crecimiento del empleo en actividades no agrícolas, así como por los fenómenos de migración campo-ciudad y hacia el extranjero, de no impulsarse políticas explícitas de fomento al desarrollo agrícola sin duda afectarán aún más la estructura primaria de gran parte de la región latinoamericana.

Una medida de esta pérdida formal se observa en la creciente importancia de productos agrícolas desde países con altas productividades en el sector primario, con fuertes apoyos públicos mediante subsidios, con alta tecnificación y capitalización del campo y gran diversidad productiva primaria agrícola, que en términos de ventajas competitivas están contribuyendo a la probable desintegración económica de ese sector como consecuencia del gran diferencial de ventajas sectoriales existentes. Asociados a esta escasa capacidad de

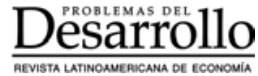


los sectores primarios están la acumulación y hegemonía de los sectores secundarios y terciarios, que utilizan y comercializan insumos y bienes importados, respectivamente, desplazando de forma progresiva productos primarios y secundarios tradicionales nacionales, los cuales van perdiendo presencia hasta ubicarse en sectores de mercado muy específico, integrados por sectores poblacionales con características de demanda también específicas y limitadas.

Sin duda, estas condiciones técnico-estructurales van en desmedro de los sectores económicos primarios de gran parte de los países latinoamericanos pero, principalmente, de la sociedad rural, que se organiza en torno a los agentes económicos y actores sociales excluidos del papel protagónico del desarrollo y reducidos a conformar segmentos tradicionales de las economías donde la relación rural-urbano mantiene características acentuadas de subordinación, sometimiento y marginación, producto del intercambio desigual con las ciudades y por la insuficiente integración del sector primario agrícola al resto de la economía.

Hacer frente a estas realidades dicotómicas requiere reformar las estructuras institucionales y promover la constitución de otras favorables al medio rural. Por ello, reconocemos que los territorios que son capaces de sustentar su desarrollo poseen un nivel institucional alto que, con frecuencia, se expresa en identidades culturales fuertes, que viabilizan sus opciones económicas endógenas y son capaces de superar las condiciones de pobreza rural. Sin embargo, en nuestros países no necesariamente se advierten atribuciones y capacidades técnicas, administrativas y políticas de los gobiernos locales, regionales, estatales y nacionales ni la necesidad de construir redes y mecanismos de comunicación entre dichos niveles de gobierno para llegar a cubrir más y mayores espacios subnacionales en su esfuerzo por promocionar el desarrollo. Hay, evidentemente, ciertos esfuerzos para revalidar los sectores rurales y considerarlos agentes activos del crecimiento y desarrollo económicos, pero aún falta mucho por aprender y hacer al respecto.

Por otra parte, habrá que señalar que pese a los esfuerzos emprendidos en los últimos años por los organismos internacionales y la mayoría de los gobiernos, aún no se logra establecer una nueva institucionalidad política que permita garantizar la sostenibilidad de procesos acumulativos de experiencias exitosas desde el enfoque territorial para el desarrollo rural. Echeverría (2003) menciona cómo, en las últimas décadas, la experiencia muestra que tanto las políticas centralizadas de desarrollo rural que no tomaron en cuenta el contexto macroeconómico, en muchos casos desfavorable, para la inversión en el ámbito rural ni los esfuerzos de promoción industrial que frenaron, directa o indirectamente, las ventajas naturales del sector agroalimentario, así como el énfasis en el crecimiento económico, ignorando la pobreza y desigualdad prevalecientes en muchas áreas de la región, fueron políticas que no dieron los resultados esperados. El agotamiento de esos esfuerzos, con la consecuente crisis de un sector público muchas veces sobredimensionado y excesi-

\section{DeSarrollo}




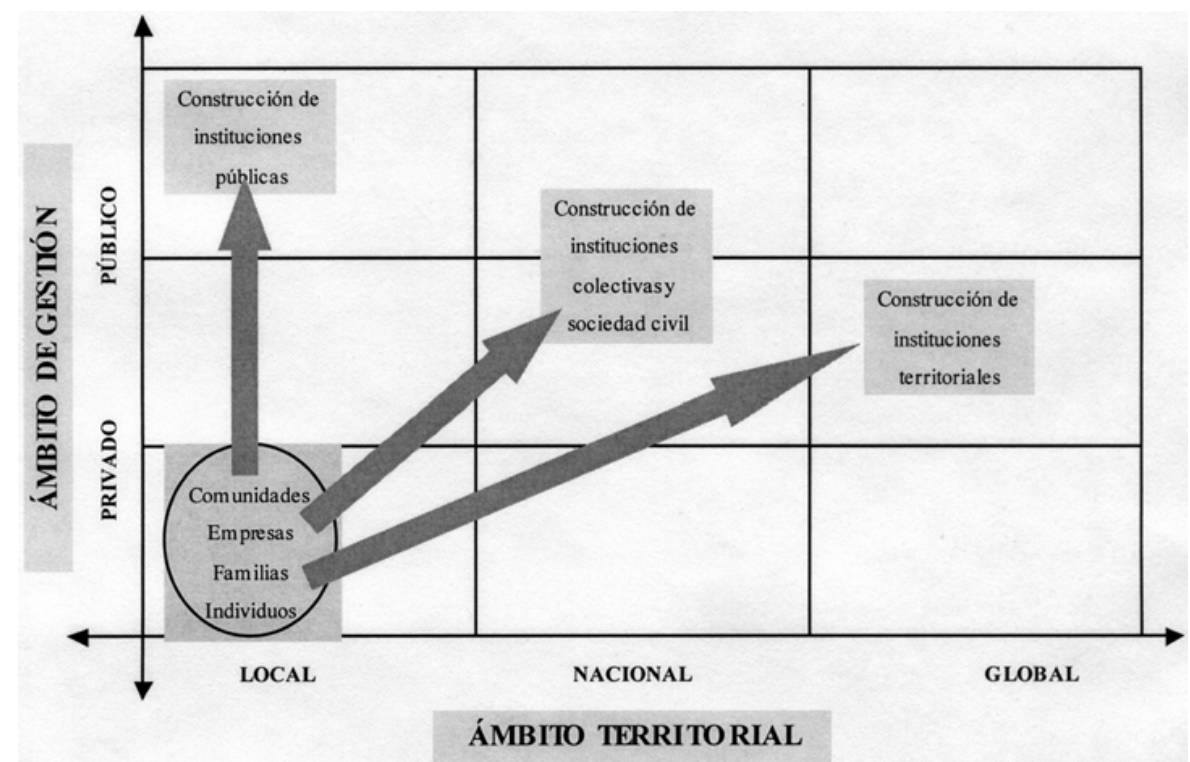

Gráfica 1. Se muestra un escenario de cambio centrado en dos dimensiones (competencias de los agentes que intervienen en el desarrollo del entorno rural): la primera, representada en el eje vertical, indica el ámbito de gestión que va de lo privado a lo público, pasando por espacios de participación corporativa local. La segunda dimensión, en el eje horizontal, indica el ámbito territorial, desde lo local hasta lo global, pasando por espacios regionales subnacionales, por lo nacional y por espacios regionales supranacionales. Las instituciones se constituyen como resultado de un proceso de acción colectiva que expresa la necesidad de asociación de los individuos, familias, empresas o comunidades, con el propósito de alcanzar sus objetivos particulares y el bien común.

Fuente: adaptado de Echeverri (2003).

vamente centralizado, ha hecho necesario que se replantee el papel del Estado en un escenario de nuevas reglas del mercado y de dimensiones específicas de las economías nacionales en el contexto de sus relaciones comerciales de carácter local e internacional. Pero, sobre todo, que asuma como responsabilidad de sus funciones un papel de árbitro y promotor, a la vez, de acciones encaminadas a reducir las asimetrías del medio rural y a favor de la equidad regional y social de sus actores. Por su parte, los otros agentes se sumarían a un proceso de cambio coordinado tomando en cuenta un ámbito de gestión colectiva y progresiva encaminada a la consolidación del ámbito territorial (Gráfica 1).

En general, esta refuncionalización de los actores públicos y civiles debe contener el objetivo de crear incentivos para lograr un uso eficiente de los recursos en el nivel macroeconómico y el aprovechamiento de las potencialidades del propio ámbito rural. Asimismo, la reorientación de las políticas debe proponer superar las ineficiencias que caracterizaron a las políticas anteriores, por medio de la descentralización, la transferencia de responsabilidades a los propios beneficiarios, el impulso a servicios de beneficio endógeno como asis-

\section{DeSarrollo}


tencia técnica e información de mercado, y la creación de asociaciones con la comunidad local.

Si bien una nueva institucionalidad de la esfera pública avanza a pasos lentos (y rezagada de la dinámica que genera las nuevas instituciones sociales), no debemos dejar de insistir en que los derroteros de esta nueva función pública debe ir ligada a un proceso de incorporación del conjunto de actores sociales rurales, fortaleciendo una visión nacional, pero de amplias ventajas para los ámbitos subnacionales y regionales, al ponderar, mediante el enfoque de desarrollo centrado en los territorios y las personas, la interacción entre territorios rurales, microrregiones, estados y municipios, que permita a los países fortalecer no sólo las unidades de producción y sus sistemas agrícolas, sino también un sistema planificado de ordenamiento territorial favorable a los procesos de cadenas productivas, aglomeraciones microrregionales, clusterización e intercambios comerciales de carácter regional, que harían de la heterogeneidad y diversidad presentes, factores de competitividad resultante de la asociación entre actores públicos y privados.

\section{Desafío ambiental y sustentabilidad en la vida rural}

El enfoque territorial del desarrollo considera también como prioridad la evaluación y promoción adecuada del uso de los sistemas naturales y ambientales a partir de una definición proactiva de sustentabilidad y en estrecha relación con los sistemas humanos con los cuales cohabitan. Esto tiene que ser así, dado que los efectos negativos del modelo neoliberal que ha predominado en las dos últimas décadas ha generado impactos devastadores en los factores tradicionales de producción y en los recursos naturales y los sistemas ecológicos que los determinan. Al respecto, Martínez Alier señala:

Las contradicciones del desarrollo neoliberal son tan profundas que hasta el desarrollo de la comunidad internacional ahora reconoce su importancia como un camino para responder a la presente crisis y buscar una ruta progresiva de transición hacia un mundo mejor. Son tan importantes, que una nueva bibliografía se está enfocando a propuestas de nivel local, incluyendo la exploración de problemas relacionados con la participación y el género, mientras que nuevas organizaciones han surgido para tomar ventaja de los espacios políticos que esta apertura está creando y para utilizar los recursos que están disponibles (Martínez Alier, 1995, citado por Barkin, 2004).

Se estima que aproximadamente 68 millones de personas en América Latina residen en ecosistemas frágiles, cifra que representa $88 \%$ de la población rural que vive actualmente en condiciones de pobreza (aproximadamente 77 millones). Echeverría (2003) precisa que la mayoría de estos pobres vive en áreas de bajo potencial agropecuario, incluyendo zonas degradadas, erosionadas o semidesérticas (como el altiplano boliviano, el chaco y el noreste de Brasil), tierras frágiles y marginales de ladera (en regiones de América Central), y humedales. Esta población depende en gran medida de los recursos naturales que 
son la base de su sustento (agua, alimento, energía e ingreso), pero por las limitantes relativas a la calidad y cantidad de estos recursos y por falta de alternativas, muchas veces cruzan el umbral de sostenibilidad y afectan, e incluso destruyen, esa base.

No obstante, comparada con otras regiones del mundo, América Latina aún goza de una amplia riqueza de recursos naturales, por lo cual los desafíos de la desertificación, la destrucción de los recursos naturales, los cambios climáticos y la pérdida de la biodiversidad deben estar cada día más presentes en la búsqueda de instrumentos de desarrollo rural sustentable y de acuerdos comunitarios incluyentes. Es por ello que tanto las convenciones internacionales (clima, biodiversidad y desertificación) como diversas estrategias impulsadas al interior de los países, señalan el interés por revertir la grave situación del deterioro ambiental: la deforestación, la degradación de los recursos hídricos y suelos, el consecuente incremento de la pobreza y la migración creciente de población rural hacia las ciudades. Pero no es suficiente enunciar los problemas y exponer públicamente un interés (generalmente acotado por frágiles acuerdos multilaterales), hoy se requiere dar fuerte impulso a acciones concretas y concertadas al interior de cada país y región a favor del medio natural, en convivencia con prácticas económicas rurales coincidentes con el desarrollo sustentable y el beneficio social de sus pobladores, tal como agentes y grupos comunitarios diversos vienen desarrollando de manera exitosa. ${ }^{5}$

El patrón de desarrollo agropecuario impulsado por las empresas trasnacionales de agronegocios más grandes ha tenido sin duda efectos negativos en el ambiente, debido a los alcances contaminantes de producciones pecuarias intensivas, al uso indiscriminado de agroquímicos, a los procesos de salinización y erosión (hídrica y eólica) que se suman al efecto devastador de la deforestación, la pérdida de bosques tropicales y de biodiversidad, lo cual ha hecho que actualmente se establezcan nuevas demandas al medio rural; entre éstas: la conservación de ecosistemas, su biodiversidad y procesos naturales esenciales; la conservación de paisajes naturales de calidad estética y biológica para soportar las actividades recreativas y alternativas económicas como el turismo rural y el ecoturismo; la producción de recursos hídricos de buena calidad para el consumo doméstico e industrial y otros servicios ambientales de creciente importancia, como el mejoramiento de los su-

5 Son destacables las experiencias del Fondo Minka de Chorlaví (cfr. Desarrollo territorial rural: Aspectos destacados de experiencias en América Latina, 2004) que apoya en países de América Latina (Bolivia, Argentina, Perú, México, Nicaragua y El Salvador) proyectos de desarrollo local, aprendizaje social comunitario, servicios ambientales, cooperativismo agroindustrial, uso y rescate de recursos hídricos y forestales, planes y programas de desarrollo microrregional sustentables, entre otros muy diversos aspectos con enfoque socio-territorial; esto permite observar un avance positivo en esta línea de preocupación del desarrollo sustentable. También son destacables experiencias en Brasil con grupos de actores rurales agrupados en el movimiento de Los sin tierra, la defensa del en torno comunitario de la mariposa monarca en México, el impulso al turismo rural cooperativista en Perú y Bolivia, la consolidación de agroempresas productoras y comercializadoras en El Salvador, y los agricultores orgánicos de Chiapas y Oaxaca en México, por mencionar algunos. 
Cuadro 4

América Latina. Tierras secas y desertificación

\begin{tabular}{|c|c|c|c|c|c|c|c|}
\hline \multirow[b]{2}{*}{ País } & \multirow{2}{*}{$\begin{array}{c}\text { Superficie } \\
\text { territorial } \\
\left(1000 \mathrm{~km}^{2}\right)\end{array}$} & \multirow{2}{*}{$\begin{array}{c}\text { Tierras } \\
\text { secas } \\
(\%)\end{array}$} & \multicolumn{3}{|c|}{ Desertificación } & \multicolumn{2}{|c|}{$\begin{array}{l}\text { Población en } \\
\text { tierras secas }\end{array}$} \\
\hline & & & $\left(1000 \mathrm{~km}^{2}\right)$ & $(\%)$ & $\begin{array}{c}\text { Población } \\
\text { (1 } 000 \text { hab.) }\end{array}$ & (1 000 hab.) & $(\%)$ \\
\hline México & 1923 & 66 & 309 & 30.5 & 100098 & 56815 & 56.9 \\
\hline Brasil & 8456 & 11 & 181 & 19.4 & 156393 & 22229 & 14.2 \\
\hline Perú & 1280 & 38 & 85 & 17.5 & 22317 & 13,583 & 60.8 \\
\hline Argentina & 2,740 & 75 & 625 & 30.5 & 35862 & 13170 & 36.7 \\
\hline Chile & 749 & 43 & 13 & 3.8 & 14000 & 7998 & 57.1 \\
\hline Bolivia & 1098 & 41 & 73 & 16.2 & 6730 & 4201 & 62.4 \\
\hline Ecuador & 277 & 27 & 5 & 6.7 & 10870 & 1990 & 18.3 \\
\hline Paraguay & 397 & 60 & 17 & 7.1 & 4391 & 69 & 1.5 \\
\hline $\begin{array}{c}\text { América Latina } \\
\text { y Caribe }\end{array}$ & 19909 & 25.3 & 1394 & 27.6 & 439684 & 121861 & 28.3 \\
\hline
\end{tabular}

Fuente: Pérez-Pardo, Octavio y Daniel Tomasini (2004), "Desarrollo rural en tierras secas", en Desarrollo territorial rural en América Latina y el Caribe: manejo sostenible de recursos naturales, acceso a tierras y finanzas rurales. Con información de: Convención de Naciones Unidas de Lucha contra la Desertificación (CNULD) (2000). CNULD, 2001. UNSO/PNUD y WRI. 1999

mideros de carbono y la emisión de oxígeno, la protección contra las inundaciones, la purificación de aguas y aire contaminados, y los equilibrios territoriales. No obstante, la discusión de soluciones a tal situación de pobreza atraviesa por el desafío de proponer medidas que involucren el contexto socioeconómico, también devastado, en que se encuentran esta área y el sector económico, lo cual es, sin duda, también un factor de degradación ambiental de raíces profundas. El desafío también reside en abordar, desde las políticas públicas, tan complejas circunstancias.

\section{Convivencia de fronteras rurales:}

\section{economía agrícola y economía rural no agrícola}

Las interacciones que tienen lugar entre la agricultura y las otras actividades económicas del medio rural son cada vez más relevantes, pero no sólo la interacción económica, tanto o más importantes son las relaciones sociales y el hecho de que todos los actores comparten un territorio cuyo patrimonio común son los recursos naturales. Otros factores como educación, salud, infraestructura y transporte, pueden incidir de manera más significativa en las condiciones de vida de los pobladores rurales, y otras actividades como el turismo, el comercio, los servicios ambientales o las industrias rurales, pueden generar ingresos equivalentes a los que genera la producción primaria.

Cuanto más al reconocer que en América Latina el medio rural abarca territorios que se conforman mayoritariamente por áreas dispersas del campo, algunas de las cuales están 
integradas a concentraciones urbanas, cuyo vínculo son los recursos naturales y productivos que muestran actualmente gran diversidad, lo que configura una amplia gama de actividades productivas interdependientes, dimensiones económicas y no económicas, y relaciones de integración funcional con lo urbano. La agricultura es y debe seguir siendo el eje articulador y, junto con ella, son diversos los factores que modifican las actuales formas organizativas del medio rural, dado que las transformaciones de esos procesos económicos han incorporado actividades que exceden a la producción agropecuaria, si bien pueden tener una clara relación con el espacio rural. También las interacciones que se dan entre territorios urbanos y rurales son hoy cada vez más frecuentes e intensas en sus dinámicas económicas, sociales y ambientales. Las migraciones de retorno, los adelantos en transportes y comunicaciones, la proliferación de instrumentos de difusión, la expansión de los mercados urbanos de los núcleos tradicionalmente concentradores de población y servicios y, en general las propias dinámicas espacio-funcionales en las cuales las ciudades juegan papeles regionales de hegemonía, están contribuyendo a la formación y construcción paulatina de estructuras diversificadas en las economías muy localizadas, desde niveles territoriales de escasa dinámica, pero que van organizándose como instrumento funcional de articulación estructural en el espacio, interactuando con centros urbanos de mayor categoría.

Por lo tanto, el desarrollo rural abarca diversas dimensiones que se complementan en el nivel territorial, como el aumento de la competitividad agroalimentaria y el manejo sostenible de los recursos naturales renovables, el desarrollo social rural, la modernización institucional y el desarrollo regional y municipal, el fortalecimiento de la infraestructura física y la integración económica subregional y regional. Si planteamos al sector agrícola en este contexto, se deberá entonces, y con mucho más énfasis que cuando se consideraba desde la concepción tradicional de ruralidad, vincularlo con los sectores no agrícolas, pero esta vez no desde los intercambios desiguales que caracterizaron históricamente los patrones de organización de los espacios divididos por jerarquías territoriales, sino aprovechando las nuevas condiciones que ofrecen las políticas macroeconómicas y sociales, para revalorar a este sector en términos de sus capacidades productivas y gerenciales, para integrar sistemas territoriales de interacción productiva, social, cultural y empresarial que dimensionen a los territorios deprimidos y con escaso crecimiento en un nuevo estatus económico.

También con el propósito de darle mayor impulso a las estrategias de desarrollo regional, los vínculos entre las economías agrícola y rural no agrícola favorecen la integración espacial, primero, al interior de los territorios rurales y, posteriormente, en su nivel de articulación estatal y nacional, lo cual permite imprimir mayor dinamismo a las regiones rurales. Dichos vínculos propician, sin duda, que éstas ya no sean vistas como agentes que compitan en situaciones muy desventajosas con otros territorios, y se aspira a que dejen 
progresivamente su papel tradicional de espacios de los cuales se extraen materias primas para incrementarles valor en los centros industriales, espacios que alimentan de mano de obra barata a las ciudades en crecimiento. Es decir, hacer que la dinámica económica que resurja en los territorios rurales contrarreste tales procesos de transferencia de valor en su forma de extracción de recursos naturales, migración e intercambios desiguales, en términos de precios de productos primarios e industriales.

Junto a ello, factores geográficos, de ubicación, tamaño y escala, y especificidad espacial, potencian las aspiraciones de las unidades territoriales por alcanzar mejores posiciones en sus indicadores sociales y económicos. El incremento progresivo de la presencia concentrada de las unidades productivas y comerciales en un espacio determinado caracterizan hoy las denominadas economías de aglomeración que la literatura reporta como promisorias para el crecimiento de los territorios. Este proceso de integralidad funcional territorial genera ventajas competitivas y, entre otros factores, hace disminuir costos de producción y facilita los procesos de transferencias como consecuencia de la diversidad sectorial y de los beneficios que comparten tanto productores, como consumidores, beneficios que no nacen del interior de las empresas, sino que se gestan fuera de éstas, lo cual hace cada vez más atractivo a un territorio para el propio capital y para la permanencia de los actores involucrados por los niveles de empleos e ingresos que ofrecen esos medios.

Para fomentar alternativas de participación entre los productores rurales es necesario que las economías de aglomeración se promuevan a escala local, que respeten las características y vocaciones de los diversos entornos territoriales rurales, las distintas escalas del capital social y la experiencia acumulada en la actividad dominante. Dichas economías deben encaminarse a privilegiar la asociación, cooperación y participación dentro de un mismo ámbito de especialización; su aplicación debe ser gradual y puede variar desde las compras en común de insumos para abaratar costos de producción, hasta comercialización a escala del producto para estar en condiciones de competir con agentes económicos de mayor experiencia. Esto puede variar también desde asociaciones para propiciar nuevas actividades vinculadas o no a la agricultura, agregación de valor mediante el fomento de labores agroindustriales, recuperación de los sistemas de cooperativas de producción con apalancamiento financiero nacional e internacional y en actividades no tradicionales que complementen y no compitan con la producción urbana regional.

Lo anterior se potencia aun más si consideramos como estrategia de participación socioeconómica local, el encadenamiento territorial y la organización espacial en redes, entendiendo por estas últimas un conjunto de elementos (desde empresas a centros de trabajo, ciudades intermedias, infraestructura, vialidades, tecnología organizacional, individuos, entre otros) interconectados de forma sistémica mediante vínculos — materiales o inmateriales- que permiten establecer flujos de intercambios — de información, conocimiento, capital, mercancías, personas—y conformar así una malla de densidad, conectividad 
y geometría variable, compuesta por nodos de distinto rango jerárquico y arcos que los entrelazan (Méndez, 2003). Según Castells, las redes constituyen la nueva morfología social de nuestras sociedades, y la difusión de su lógica de enlace modifica la operación y los resultados de los procesos de producción, la experiencia, el poder y la cultura. La presencia o ausencia en la red, y la dinámica de cada una frente al resto, son fuentes cruciales de dominio y cambio en nuestra sociedad, una sociedad que, por lo tanto, puede llamarse con propiedad la sociedad red (Castells, 1997). Hay quienes sugieren que esos procesos son totalmente ajenos al mundo rural de América Latina, pero autores como Alburquerque (2004) demuestran lo contrario.

Las cadenas territoriales pueden surgir a partir de las capacidades que tengan los actores económicos y las empresas para vincularse con otros lugares, o según su capacidad de construcción de redes u organizaciones dentro del mismo territorio, junto a las capacidades de gestión pública que acompañen la organización de esos procesos. Silva (2003) indica que esas últimas capacidades están íntimamente relacionadas con la idea de construcción social de los territorios y, más concretamente, con la idea de construcción de territorios innovadores y competitivos en la medida en que la estructura económica nacional se pueda expresar en torno a cadenas productivas locales que propicien el desarrollo de las pequeñas y medianas empresas y tiendan a buscar formas asociativas y articuladas para conseguir ventajas competitivas, ya sea bajo el impulso de aglomeraciones de empresas organizadas en el territorio o de otros tipos de asociaciones productivas en las cuales el logro de economías de escala se conciben como externas a las empresas pero internas a los territorios.

Sin embargo, habría que acotar que debido a la dinámica, tamaño y dimensiones socioeconómicas de los territorios rurales, tomando en cuenta la escala de los impactos favorables posibles de impulsar, las economías de aglomeración en el entorno rural no deben buscar irradiaciones territoriales amplias, sino resolver problemas de ocupación, demanda y competitividad local en una realidad de mercado. Se trata de ver a cada unidad campesina o rural como una forma de organización específica en una estrategia integrada de desarrollo sustentable y en un esquema flexible de aglomeración económica, en una dimensión empresarial susceptible de organizarse, competir, capitalizarse y construir una nueva estructura rural mediante lineamientos económicos, pero con distribución equitativa de beneficios socialmente retribuibles. Quizá éste es el mejor medio para consolidar procesos de integración social y territorial.

Otro tema relevante tiene que ver con la importancia de los procesos de industrialización rural en contextos amplios y heterogéneos, dado que se asocian tanto con la acumulación progresiva de capital, incrementos de niveles de empleo, valores agregados de productos primarios agrícolas (agroindustria) y productos no primarios (intermedios), así como con la integración sectorial en la estructura económica local, lo cual puede construir 
procesos de cambios estructurales capaces de organizar entornos adecuados de crecimiento territorial en áreas rurales.

Desde el punto de vista de las medidas que inducen el desarrollo rural, los retos del crecimiento territorial en estas áreas son, sin embargo, complicados de lograr si no se tiene una visión certera de las dinámicas territoriales que podrían ofrecer ciertas ventajas para la organización primaria de esta industrialización, pero que, sin embargo, de acuerdo con las características de los espacios rurales tradicionales, están expuestos a importantes efectos externos adversos por razones de los diferenciales en tasas de acumulación, relaciones capital-trabajo, tasa de innovación y cambio técnico, y ritmos espontáneos de competitividad territorial con otros espacios. Sin embargo, frente a esto último, los territorios rurales poseen importantes ventajas comparativas, pero no ya desde las tradicionales formas de abordar dichas ventajas, que son la presencia y cercanía a los recursos naturales y obtención de bienes primarios, sino desde el nivel de conocimiento que tienen de su entorno y de sus grados y capacidades de organización y articulación social desde los lazos culturales y de arraigo que facilitan la construcción de redes sociales. Ello es propicio para la creación de grupos de productores que, organizados en torno a procesos de industrialización, pudieran fortalecer conjuntos de empresas organizadas espacialmente a manera de cluster, en las cuales la externalidad más importante son los lazos culturales y la tradición de trabajo colectivo, así como los conocimientos transmitidos intergeneracionalmente. Otro ejemplo representativo serían los conglomerados existentes alrededor de una cadena agroalimentaria-agroindustrial constituidos por empresas agroindustriales, proveedoras de insumos y de servicios, además de muchas organizaciones cuyo quehacer influye de manera decisiva en la competitividad de la agricultura y de la industria rural.

Es por ello que la industrialización rural ha sido una aspiración en diversos gobiernos de países latinoamericanos, al igual que de los planificadores del territorio. Se piensa que dicha alternativa sirve para alcanzar mejor equilibrio entre los ámbitos rural y urbano. Sin embargo, los proyectos han fracasado invariablemente tanto por el tipo de estrategia que se adopta, como por el descuido del contexto en el cual se aplica. En el primer caso se ha intentado desarrollar distritos industriales que se enfrentan a carencias de infraestructura y presentan muy limitada irradiación de beneficios locales; en el segundo, el tipo de industrialización seguido no corresponde con las vocaciones productivas del territorio, la experiencia acumulada o el nivel de desarrollo del contexto en el cual se instrumenta.

Por lo tanto, impulsar un redimensionamiento del sector agrícola y una nueva estrategia de industrialización rural, junto con el desarrollo de clusters territoriales, debe tomar en cuenta los avances recientes de la organización del trabajo, la modernización de la economía, las dinámicas de las demandas de los mercados internacionales para la integración de nichos alternativos competitivos y el desarrollo de nuevas modalidades para desarrollar actividades diversificadas. En tal contexto, un programa de industrialización rural

\section{DeSarrollo}


debe partir de la vocación y potencialidad del territorio encaminado al fomento de la competitividad en condiciones reales y, de esa manera, acceder a nichos de mercado local, regional, nacional e internacional. Es aquí donde el concepto de competitividad sistémica potencia las capacidades locales y permite dimensionar los alcances de largo plazo que los programas de desarrollo pudieran considerar para cada territorio específico. También deben tomarse en cuenta, por lo menos, las siguientes necesidades: $a$ ) mejorar la tecnología de producción; $b$ ) considerar el medio ambiente, y $c$ ) someterse a regulaciones y supervisiones, ya sea por organismos o redes de autocontrol, o por otros externos, de supervisión y certificación.

En fin, el despliegue de capacidades productivas de la población rural resulta indispensable para hacer emerger un proyecto rural con un fortalecimiento de encadenamientos territoriales y con un enfoque sostenible y de estricto cuidado ambiental.

\section{Reflexiones finales}

Recapitulando en torno a las dimensiones territoriales que debe contener el desarrollo rural en la actualidad, a continuación se enuncian algunas propuestas finales orientadas a reconvertir el papel de las políticas públicas y del Estado, en otra función que se responsabilice del desarrollo a favor de la población y la vida rural.

1) A lo largo del texto se ha insistido en la idea central de que el papel del medio rural es fundamental en las estrategias de desarrollo económico de los países latinoamericanos y que los desafíos sociales de sus pobladores debe representar el objetivo de atención principal. En ese contexto, el enfoque territorial del desarrollo se propone implantar acciones que incidan en el reforzamiento institucional, la organización y promoción de los territorios rurales desfavorecidos, el fortalecimiento de las actividades agropecuarias y la coadyuvancia en el desarrollo de capacidades técnicas, administrativas, de organización social y producción locales.

2) La relación estrecha entre territorialidad y políticas públicas obliga a asignar al Estado un papel preponderante en las estrategias de planeación territorial del medio rural y considerar que las políticas macroeconómicas, en lo específico, deben formularse a partir ya no sólo de la distribución espacial de indicadores económicos agregados, sino también del reconocimiento de las capacidades locales y las diferencias territoriales existentes, con la finalidad de promover dinámicas económicas internas. En ese sentido, las políticas macroeconómicas deberían orientarse a promover estrategias de desarrollo económico con medidas territorializadas (políticas de desarrollo regional o local), considerando a los actores locales como emergentes mediante una nueva institucionalidad, con gran capacidad para promover y participar en el nuevo contexto. La participación ciudadana se postula, desde esta perspectiva, como elemento clave en el proceso de transformación.

\section{DeSarrollo}


3) Los territorios rurales se complementan, lo cual favorece su funcionalidad. La mejor forma de garantizar interfuncionalidades territoriales es por medio de la construcción o consolidación de redes diversas (sociales, productivas, empresariales, políticas, entre otras), que interaccionen con los mecanismos expeditos de comunicación e interfuncionalidad de los distintos niveles de gobierno: local, regional, estatal o nacional, con los agentes locales de desarrollo, en los cuales se considere la integración espacial en relación con un mayor número de comunidades y territorios interactuando entre sí, y generando competitividad a partir de economías de escala territoriales.

4) Hay elementos estructurales en la formulación de políticas públicas que debieran cambiar de manera radical para hacer frente a la situación de pobreza, desintegración y abandono del ámbito rural. Por ello se propone cambiar el énfasis de los enfoques de desarrollo, de uno que prioriza el crecimiento sin tomar en cuenta en qué territorios se genera, a otro en el cual el bienestar se dé sobre la base de la dinámica local como garantía de futuras redistribuciones sociales. La equidad social sería la fortaleza de la nueva institucionalidad territorial.

5) Las políticas centralizadas - e incluso las políticas rurales también centralizadas que le apostaron al crecimiento en un cierto sector económico y en unas cuantas ciudades y territorios - deben cambiar a favor de la integración sectorial y territorial en condiciones de complementariedad, junto a la aplicación de políticas social y territorialmente diferenciadas. Para ello se propone garantizar la complementariedad intersectorial, promocionando elementos comparativos y de competencia sistémica, tanto natural, como sociocultural y productiva del conjunto de territorios, al mismo tiempo que impulsa procesos de descentralización y transferencia de responsabilidades a los actores localesrurales, con el fin de crear asociaciones formales con las comunidades locales y los agentes de desarrollo.

6) Los conflictos sociales y las desarticulaciones socio-territoriales originados por factores diversos, como los desplazamientos migratorios y la permanente marginación, constituyen una realidad que hace más complicado proponer acciones de mejora sustancial para el medio rural. Sin embargo, la gestión pública debe enfocar sus políticas aceptando una nueva corresponsabilidad para con esos procesos y desplegar estrategias de desarrollo asociadas con los activos locales y la actividad económica tradicional de las comunidades. Para ello es indispensable restituir el papel integrador de la agricultura, la agroindustria y los agronegocios al interior de los sistemas territoriales; respetar los intereses locales, culturales y de tradiciones que favorecen la integración comunitaria; dimensionar favorablemente las actividades de la economía rural no agrícola; fortalecer procesos encaminados a consolidar cadenas productivas y cadenas interterritoriales, que sean al mismo tiempo socialmente redituables al territorio rural.

\section{DeSarrollo}


7) Finalmente, la gestión pública en beneficio de los territorios rurales debe ser promotora de programas de desarrollo con un carácter de multisectorialidad operativa, fortalecer la visión de comunidad como eje fundamental de integración rural y promover una vida digna y reconstitutiva de las tradiciones y las culturas populares con la premisa de que el desarrollo sustentable es posible.

Bibliografía

Alburquerque, Francisco, Desarrollo económico local y descentralización en América Latina: un análisis comparativo, Santiago de Chile, CEPAL y Sociedad Alemana de Cooperación Técnica (GTZ), 2004.

aregional.com, Índice de competitividad sistémica de las entidades federativas, México, 2005.

Banco Interamericano de Desarrollo (BID), Latin American Labor Markets in the 1990s: Deciphering the Decade, primer Seminario Técnico de Consulta Regional sobre Temas Laborales, Panamá, 2001.

Banco Mundial, Llegando a los pobres de las zonas rurales, estrategia de desarrollo rural para América Latina y el Caribe, Washington, 2002.

Barkin, David, "Superando el paradigma neoliberal: desarrollo popular sustentable", en Norma Giarracca (compiladora), ¿Una nueva ruralidad en América Latina?, Buenos Aires, ClACso, 2001.

-, "Forjando una estrategia alternativa en México para aprovechar el comercio mundial", en Cuadernos del CENDES, año 21, núm. 55, eneroabril, Caracas, 2004.

Carpio Martín, José, "Desarrollo local en los espacios rurales", Madrid, Universidad Complutense, 2002.

Castells, Manuel, La era de la información. Economía, sociedad y cultura, vol. 1: La sociedad Red, Madrid, Alianza, 1997.

CEPAL, Anuario estadístico de América Latina y el Caribe, Santiago de Chile, 2004.

- Panorama social de América Latina, 20032004, Santiago de Chile, 2005.

CEPAL-FAO, Empleo e ingresos rurales no agrícolas en América Latina, Santiago de Chile, 2004.

Convención de Naciones Unidas de Lucha contra la Desertificación (CNULD), Evaluación del estado de degradación de las tierras en las zonas áridas, semiáridas y subhúmedas secas. Informe presentado ante la Iv Conferencia de las Partes, Secretaría de la Convención, http://www.unccd. int/php/document.php? $r e f=I C C D / C O P(4) / I n f .6$, 2000.
—- Desertificación y sequía en América Latina y el Caribe, Unidad ALyC, Secretaría de la CNULD, Bonn, mimeo, 2001.

Cordero-Salas, Paula; H. Chavarría, R. Echeverri y S. Sepúlveda, Territorios rurales, competitividad y desarrollo, San José, Instituto Interamericano de Cooperación para la Agricultura (IICA), 2003.

Chavarría, Hugo; Sergio Sepúlveda y Patricia Rojas (compiladores), Competitividad: cadenas agroalimentarias y territorios rurales. Elementos conceptuales, San José, IICA, 2002.

Delgadillo, Javier (coordinador), El desarrollo territorial en Tlaxcala: dimensiones rurales y opciones de ordenamiento espacial en la Región Oriente, México, conacyt, El Colegio de Tlaxcala, 2005.

- (coordinador), Enfoque territorial para el desarrollo rural en México, México, unam, 2005.

De Janvry, A. y E. Saudolet, Household Modeling for the Design of Poverty Alleviation Strategies, Working Paper núm. 787, Department of Agricultural and Resource Economics, Univerisity of California at Berkeley, 1996.

Dirven, Martin, Alcanzando las metas del milenio: una mirada hacia la pobreza rural y agrícola, Santiago de Chile, CEPAL, Red de Desarrollo Agropecuario, 2004.

Echeverri, Rafael y María P. Rivero, Nueva ruralidad. Visión del territorio en América Latina y el Caribe, San José, IICA, 2002.

Echeverri, Rafael, La armonización del desarrollo rural con el desarrollo económico: Soluciones globales o soluciones regionales, San José, IICA, 2003.

Echeverría, Rubén (editor), Desarrollo territorial rural en América Latina y el Caribe: manejo sostenible de recursos naturales, acceso a tierras y finanzas rurales, Washington, BID, 2003.

Escobal, Javier; Jaime Saavedra y Máximo Torero, Los activos de los pobres en el Perú, Lima, GRADE, 1998, documento de trabajo, 26, 1998.

Escobal, Javier y Carmen Ponce, "Innovaciones en la lucha contra la pobreza rural en América

\section{DeSarrollo}


Latina", documento preparado para la CEPAL, presentado en el Encuentro de Altos Directivos contra la Pobreza Rural, Santiago de Chile, 27 y 28 de enero de 2000.

FAO (Organización de las Naciones Unidas para al Agricultura y la Alimentación), Assessment of the World Food Security Situation, Committee on World Food Security, $28^{\text {th }}$ Session, Roma, 68 de junio, en http://www.fao.org/unfao/bodies/ cfs/cfs28/CFS2002-e.htm, 2002.

Fondo Mink'a de Chorlaví, Desarrollo territorial rural: Aspectos destacados de experiencias en proceso en América Latina, Santiago de Chile, RIMISP, 2002.

Foro Económico Mundial, Índice de sostenibilidad ambiental 2002, Foro Económico Mundial, Yale Center for Environmental Law and Policy (Yale University), Center for International Earth Science Information Network (Columbia University), 2003.

Giarracca, Norma (compilador), ¿Una nueva ruralidad en América Latina?, Buenos Aires, CLACSO, 2001.

Instituto Interamericano de Cooperación para la Agricultura (IICA), La contribución de la agricultura a la redistribución de la pobreza, a la creación de empleo y a la prosperidad rural, documento en construcción, San José, 2005.

_- Situación y perspectivas de la agriculturay de la vida rural en las Américas, San José, 2004.

,"Capital social, institucionalidad y territorios”, en Sinopsis, núm. 5, mayo, San José, 2003.

Köbrich, Claus; Liliana Villanueva y Martin Dirven, Pobreza rural y agricola: entre los activos, las oportunidades y las políticas, Una mirada hacia Chile, Santiago de Chile, Unidad de Desarrollo Agrícola, CEPAL, 2004.

Martínez Alier, Juan, De la economía ecológica al ecologismo popular, Barcelona, Icaria, 1995

Méndez Gutiérrez del Valle, Ricardo, "Globalización, redes y nuevos contrastes territoriales", en $\mathrm{M}$. Valenzuela (coordinador), Un mundo por descru-brir en el siglo XXI, Madrid, Real Sociedad Geográfica, 2003.

PNUD, Informe de desarrollo humano 2003, Nueva York, 2004.
Reardon, T., "Agroindustrialization in Intermediate Cities in Latin America: Hypotheses Rregarding Employment Eeffects on the Rural Poor", presentado al IFPRI Workshop on Strategies for Stimulating Growth of the Rural non Farm Economy, 2004.

Reardon, Thomas; Julio Berdegué y Germán Escobar, "Empleo e ingresos rurales no agrícolas en América Latina: Síntesis de implicaciones de políticas", en CEPAL-FAO, Empleo e ingresos rurales no agrícolas en América Latina, Santiago de Chile, 2004.

Rodríguez González, Román y Edelmira Pérez Correa, El desarrollo rural como paradigma teórico", Asturias, Trea, 2004.

Schejtman y Berdegué, Desarrollo territorial rural, Santiago de Chile, RIMISP, 2003.

Sepúlveda, Sergio; A. Rodríguez, R. Echeverri y M. Portilla, El enfoque territorial del desarrollo rural, San José, IICA, Dirección de Desarrollo Rural Sostenible, 2003a.

- Territorio rurales, estrategias y políticas en América Latina, San José, IICA, 2003b.

Silva L., Iván, Disparidades, competitividad territorial y desarrollo local y regional en América Latina, Santiago de Chile, ILPES- CEPAL, 2003.

Soja, E., The Political Organization of Space, Washington, Association of American Geographers, 1971.

Transparencia Internacional, Índice de percepción de la corrupción 2002, 2003.

Trejos, Rafael A.; Carlos Pomareda y Juan Villasuso, Políticas e instituciones para la agricultura de cara al siglo XXI, San José, IICA, 2004.

UNSO/PNUD y WRI, Drylands Population Assessment II, sitio Web de la Oficina de PNUD para la Lucha contra la Desertificación, http://www.undp.org/ seed/unso/pub-htm/dryland-population.pdf 1999.

Van Hemelryck, Libero, "El enfoque sistémico del desarrollo local", en Enfoque sistémico y desarrollo local (manual), Escuela de Planificadores Sociales, 2005.

\section{Desarrollo}

